\title{
Near-Pseudoinverse Control of Kinematically Redundant Manipulators With the Constraint of Repeatability
}

Rodney G. Roberts

Anthony A. Maciejewski

School of Electrical Engineering

Purdue University

West Lafayette, Indiana $\mathbf{4 7 9 0 7}$

\begin{abstract}
The issue of repeatability is addressed for a particular redundant manipulator. This particular manipulator is a planar three-link manipulator with two orthogonal, prismatic joints and a third revolute joint. This manipulator can be thought of as a mobile robot with one revolute joint. It is shown that pseudoinverse control has no stable surfaces for this robotic manipulator. A class of repeatable inverses is then derived. Finally the repeatable inverse which is closest in an integral norm sense to the pseudoinverse is found from this class.
\end{abstract}

\section{Introduction}

It is a well known fact [2] that the pseudoinverse solution for a kinematically redundant manipulator is in general nonconservative. In other words, pseudoinverse control of kinematically redundant manipulators produces a drift in joint space when a cyclic task is performed in rectilinear space. Klein and Kee [3] showed that this drift has predictable properties including a numerically stable limit in some situations. If such a limit exists, the appropriate initial joint values could be set to guarantee conservative solutions. Shamir and Yomdin [5] have shown that for some manipulators there are areas in the joint space in which the pseudoinverse is repeatable. They established a necessary condition for such an integral surface to exist. This condition requires that the Lie bracket of any two columns of the inverse be a linear combination of the columns of the inverse on the particular surface in question. The Lie bracket of two vectors $u$ and $\mathbf{v}$, where both vectors are functions of $\theta$, is given by

$$
[\mathbf{u}, \mathbf{v}]=\left(\frac{\partial \mathbf{v}}{\partial \theta}\right) \mathbf{u}-\left(\frac{\partial \mathbf{u}}{\partial \theta}\right) \mathbf{v}
$$

If it turns out that this surface is in fact an integral surface, then a choice of initial joint settings which lies on this surface will guarantee repeatability. An integral surface is stable in the sense that if the manipulator starts on the surface then it will continue to stay on that surface. An example is given in [5] where the stable surfaces for the pseudoinverse solution of the absolute joint angles of a three-link planar manipulator are calculated. If an initial joint value is not on one of these stable surfaces then the pseudoinverse control may cause it to approach a stable surface. Baillieul [1] has derived a class of repeatable inverses, called the extended Jacobians, which actually have a foliation of stable surfaces.

\section{Nonexistence of Stable Surfaces}

It can be shown that pseudoinverse control for many types of manipulators may not have any stable surfaces [4]. In particular, consider the planar manipulator with two orthogonal prismatic joints and a third revolute joint of unit length shown in Fig. 1. The kinematic function has the form

$$
\mathbf{x}=\mathbf{f}(\theta)=\left[\begin{array}{l}
d_{1}+\cos \theta_{3} \\
d_{2}+\sin \theta_{3}
\end{array}\right]
$$

where $\mathbf{x}=[x, y]^{T}$ and $\theta=\left[d_{1}, d_{2}, \theta_{3}\right]^{T}$. For this manipulator the Jacobian equation is given by

$$
\dot{\mathbf{x}}=J \dot{\theta}
$$

where

$$
J=\left[\begin{array}{ccc}
1 & 0 & -\sin \theta_{3} \\
0 & 1 & \cos \theta_{3}
\end{array}\right] .
$$

Since $J$ in this case is clearly always of full row rank, the pseudoinverse can be calculated using $J^{T}\left(J J^{T}\right)^{-1}$ which yields

$$
J^{+}=\frac{1}{2}\left[\begin{array}{cc}
1+\cos ^{2} \theta_{3} & \sin \theta_{3} \cos \theta_{3} \\
\sin \theta_{3} \cos \theta_{3} & 1+\sin ^{2} \theta_{3} \\
-\sin \theta_{3} & \cos \theta_{3}
\end{array}\right]
$$

Let $k_{1}$ and $k_{2}$ be the first and second columns of this inverse, respectively. Then the Lie bracket of $\mathbf{k}_{1}$ and $\mathbf{k}_{\mathbf{2}}$ is given by

$$
\left[\mathbf{k}_{1}, \mathbf{k}_{2}\right]=\frac{1}{4}\left[\begin{array}{r}
\sin \theta_{3} \\
-\cos \theta_{3} \\
1
\end{array}\right]
$$


It can easily be seen that $\left[k_{1}, k_{2}\right]$ is orthogonal to $k_{1}$ and $\mathbf{k}_{2}$ so that $\left[\mathbf{k}_{1}, \mathbf{k}_{2}\right]$ cannot be a linear combination of $\mathbf{k}_{1}$ and $\mathbf{k}_{2}$. This result violates the necessary condition for the existence of an integral surface.

One could also use the method described in [2] to show the nonconservativeness of the pseudoinverse for this particular manipulator. Consider the following Pfaffian differential equation:

$$
\mathbf{n}_{\mathbf{J}} \cdot \mathbf{d} \theta_{\mathbf{P}}=\mathbf{0}
$$

where $n_{J}$ is a homogeneous solution of (3) and $d \theta_{p}$ is the pseudoinverse solution for $d \theta$. The null space of $J$ can be described by

$$
\mathbf{n}_{\mathbf{J}}=\alpha\left[\begin{array}{r}
\sin \theta_{3} \\
-\cos \theta_{3} \\
1
\end{array}\right]
$$

where $\alpha$ is a nonzero scalar. A necessary and sufficient condition for ( 7 ) to be integrable is that

$$
\mathbf{n}_{\mathbf{J}} \cdot \boldsymbol{\nabla} \times \mathbf{n}_{\mathbf{J}}=\mathbf{0} .
$$

However, evaluating this expression using (8) results in $\mathbf{n}_{\mathbf{J}} \cdot \nabla \times \mathbf{n}_{\mathbf{J}}=-\boldsymbol{\alpha}^{2}$ which once again proves that there are no integral curves for this manipulator when using pseudoinverse control.

\section{A Class of Repeatable Inverses}

The inverses under consideration are generalized inverses relating the cartesian velocity of the end effector to the joint velocities of the manipulator by the equation

$$
\dot{\theta}=K \dot{\mathbf{x}}
$$

where $J K=I$. Any inverse $K$, for a manipulator with a single degree of redundancy, can be written in the form

$$
K=J^{+}+\hat{\mathbf{n}}_{J} \mathbf{w}^{T}
$$

where $\hat{\mathbf{n}}_{J}$ is the unit length null vector of the Jacobian and $w$ is a vector which uniquely determines $K$. For the manipulator in Fig. $1 \hat{\mathbf{n}}_{J}$ is given by

$$
\hat{\mathbf{n}}_{J}=\frac{1}{\sqrt{2}}\left[\begin{array}{c}
\sin \theta_{3} \\
-\cos \theta_{3} \\
1
\end{array}\right]
$$

By properly selecting $w$ the inverse $K$ can be made to be repeatable. When dealing with the question of repeatability for three-link planar manipulators, it is easier to consider the "curl" criterion rather than the equivalent Lie bracket condition described in [5]. This curl criterion is given below:
Theorem The Pfaffian $X \cdot d x$ is integrable if and only if

$$
\boldsymbol{X} \cdot \boldsymbol{\nabla} \times \boldsymbol{X}=\mathbf{0} .
$$

Proof: See [6].

For the application at hand this becomes

$$
\mathbf{n}_{K} \cdot \nabla \times \mathbf{n}_{K}=\mathbf{0}
$$

where $\mathbf{n}_{K}$ is the null vector of $K^{T}$. From equation (11) it is easy to verify that

$$
\mathbf{n}_{K}=J^{T} \mathbf{w}-\hat{\mathbf{n}}_{J} .
$$

Thus for this particular case

$$
\mathbf{n}_{K}=\left[\begin{array}{c}
w_{1}-\frac{1}{\sqrt{2}} \sin \theta_{3} \\
w_{2}+\frac{1}{\sqrt{2}} \cos \theta_{3} \\
-w_{1} \sin \theta_{3}+w_{2} \cos \theta_{3}-\frac{1}{\sqrt{2}}
\end{array}\right] .
$$

Before substituting (16) into (14), the following substitutions are made

$$
\begin{aligned}
& \gamma=w_{1}-\frac{1}{\sqrt{2}} \sin \theta_{3} \\
& \eta=w_{2}+\frac{1}{\sqrt{2}} \cos \theta_{3}
\end{aligned}
$$

which results in

$$
\mathbf{n}_{K}=\left[\begin{array}{lll}
\gamma & \eta & -\gamma \sin \theta_{3}+\eta \cos \theta_{3}-\sqrt{2}
\end{array}\right]^{T} .
$$

For the case of a mobile robot, it is desirable to make the inverse only a function of $\theta_{3}$ so that $w_{1}$ and $w_{2}$ are just functions of $\theta_{3}$. Taking the curl of equation (19) gives

$$
\nabla \times \mathbf{n}_{K}=\left[\begin{array}{lll}
-\eta^{\prime} & \gamma^{\prime} & 0
\end{array}\right]^{T}
$$

where' denotes differentiation with respect to $\theta_{3}$. Thus equation (14) which characterizes all repeatable inverses which are functions of $\theta_{3}$ becomes

$$
\mathbf{n}_{K} \cdot \nabla \times \mathbf{n}_{K}=-\gamma \eta^{\prime}+\eta \gamma^{\prime}=0 .
$$

Solving this ordinary differential equation gives the solution

$$
\gamma=k \eta
$$

where $k$ is a constant. Substituting equations (17) and (18) into equation (22) yields

$$
w_{1}=k w_{2}+\frac{1}{\sqrt{2}}\left(\sin \theta_{3}+k \cos \theta_{3}\right)
$$


which parametrizes the set of repeatable solutions in terms of the function $w_{2}$ and the constant $k$.

\section{Optimization}

Now that a class of repeatable inverses has been derived, the optimal member of this class with respect to an appropriately chosen criterion function can be found. Let this criterion function be given by

$$
C(k)=\int_{a}^{b}\left\|K-J^{+}\right\|_{2}^{2} d \theta_{3} .
$$

This criterion function measures the distance of the inverse $K$ from the pseudoinverse in the region $a \leq$ $\theta \leq b$ in an integral norm sense. It can be easily shown that

$$
\left\|K-J^{+}\right\|_{2}^{2}=\|\mathrm{w}\|_{2}^{2}=w_{1}^{2}+w_{2}^{2} .
$$

It is thus important to minimize $w_{1}^{2}+w_{2}^{2}$. Using equation (23) and completing the square gives

$$
\begin{aligned}
w_{1}^{2}+w_{2}^{2}= & \left(k^{2}+1\right)\left[w_{2}+\frac{k\left(\sin \theta_{3}+k \cos \theta_{3}\right)}{\sqrt{2}\left(k^{2}+1\right)}\right]^{2} \\
& +\frac{\left(\sin \theta_{3}+k \cos \theta_{3}\right)^{2}}{2\left(k^{2}+1\right)}
\end{aligned}
$$

Now there is nothing that can be done about the last term of equation (26); however the first term can be minimized by setting

$$
w_{2}=-\frac{k\left(\sin \theta_{3}+k \cos \theta_{3}\right)}{\sqrt{2}\left(k^{2}+1\right)}
$$

which from (23) results in

$$
w_{1}=\frac{k \cos \theta_{3}+\sin \theta_{3}}{\sqrt{2}\left(k^{2}+1\right)} .
$$

Equations (27) and (28) give the minimal solution for a repeatable inverse as a parametrization of $k$.

The cost function in equation (24) when evaluated over an interval of length $2 \pi$ has a value of $\pi / 4$ for all $k$ so that all choices of $k$ result in the same cost. However most manipulators have joint limits and one may only want to integrate over those joint values which are obtainable by the manipulator. Suppose that the range of values for $\theta_{3}$ is from $a$ to $b$ where $-\pi \leq a<b \leq \pi$. Then $C(k)$ is given by

$$
C(k)=\int_{a}^{b} \frac{\left(\sin \theta_{3}+k \cos \theta_{3}\right)^{2}}{2\left(k^{2}+1\right)} d \theta_{3} .
$$

After some calculation one sees that

$$
C(k)=\frac{C_{1} k+C_{2}}{2\left(k^{2}+1\right)}+C_{3}
$$

where

$$
\begin{gathered}
C_{1}=\int_{a}^{b} \sin 2 \theta_{3} d \theta_{3}=\frac{\cos 2 a-\cos 2 b}{2} \\
C_{2}=-\int_{a}^{b} \cos 2 \theta_{3} d \theta_{3}=\frac{\sin 2 a-\sin 2 b}{2}
\end{gathered}
$$

and

$$
C_{3}=\frac{1}{2} \int_{a}^{b} \cos ^{2} \theta_{3} d \theta_{3}=\frac{b-a-C_{2}}{4} .
$$

It can be easily shown that if $C_{1}$ is nonzero the optimal choice for $k$ is

$$
k=\frac{C_{2}-\sqrt{C_{1}^{2}+C_{2}^{2}}}{C_{1}} .
$$

When $C_{1}=0$ and $C_{2}<0$ the minimum will occur when $k=0$. When $C_{1}=0$ but $C_{2}>0$ the value of $C(k)$ will approach zero as $k$ approaches $\pm \infty$. Finally, when $C_{1}$ and $C_{2}$ are both zero as in the case of integrating on $[-\pi, \pi]$, then any $k$ will give the same value for $C(k)$.

For the case of symmetric joint limits, i.e. when $a=-b$, the optimal choice for $k$ breaks down into special cases since $C_{1}$ is always zero. These cases are summarized in Table 1 . When $0<b<\pi / 2$ the inverse $K$ differs from the pseudoinverse only in the first column while for $\pi / 2<b<\pi$ the difference is only in the second column. When $b$ takes on the values $\pi / 2$ and $\pi$ any value of $k$ will result in a minimal inverse.

\section{Summary}

The nonexistence of stable surfaces for the pseudoinverse control of a 2-P-1-R robot manipulator was shown. The class of repeatable inverses which are functions of the angle of the revolute link of the manipulator was derived. The inverse in this class of repeatable inverses which is closest to the pseudoinverse in an integral norm sense was found.

\section{References}

[1] Baillieul, J., "Kinematic Programming Alternatives for Redundant Manipulators," in Proceedings of the International Conferences on Robotics and Automation, St. Louis, MO, March 1985, pp. 722-728.

[2] Klein, C.A. and C.H. Huang, "Review of Pseudoinverse Control for Use with Kinematically Redundant Manipulators," IEEE Trans. on Systems, Man, and Cybernetics, Vol. SMC-13, No. 3, March/April 1983, pp. 245-250. 
[3] Klein, C.A. and K.H. Kee, "The Nature of Drift in Pseudoinverse Control of Kinematically Redundant Manipulators," IEEE Trans. on Robotics and Automation, Vol. 5, No. 2, April 1989, pp. 231-234.

[4] Maciejewski, A.A. and R.G. Roberts, "Utilizing Kinematic Redundancy in Robotic Systems: Practical Implementations and Fundamental Limitations," American Controls Conference, San Diego, CA, May 23-25, 1990, pp. 209-214.
(5) Shamir, T. and Y. Yomdin, "Repeatability of Redundant Manipulators: Mathematical Solution of the Problem," IEEE Trans. on Automatic Control, Vol. 33, No. 11, November 1988, pp. 1004-1009.

[6] Sneddon, I.N., Elements of Partial Differential Equations, New York: McGraw Hill, 1957.

Fig. 1 Geometry of a planar three-link manipulator whose first two joints are prismatic and whose last joint is revolute and of unit length.

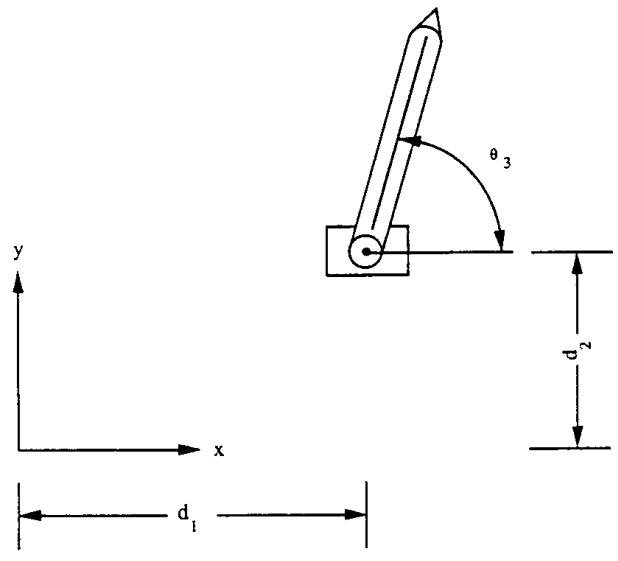

Table 1. Optimal Parameters for Symmetric Joint Limits $\left(C_{1}=0\right)$

\begin{tabular}{|c|c|c|c|c|}
\hline & $0<b<\frac{\pi}{2}$ & $b=\frac{\pi}{2}$ & $\frac{\pi}{2}<b<\pi$ & $b=\pi$ \\
\hline$C_{2}$ & - & 0 & + & 0 \\
$k_{\text {opt }}$ & 0 & any value & $\infty$ & any value \\
$w_{1}$ & $\frac{1}{\sqrt{2}} \sin \theta_{3}$ & Eq.(28) & 0 & Eq.(28) \\
$w_{2}$ & 0 & Eq.(27) & $-\frac{1}{\sqrt{2}} \cos \theta_{3}$ & Eq.(27) \\
\hline
\end{tabular}

